\title{
THE CHECKLIST OF LIVERWORTS (Hepaticae) AND HORNWORTS (ANTHOCEROTAE) OF VIETNAM UPDATED BASED ON LITERATURE SURVEY
}

\author{
Vadim Bakalin ${ }^{1 *}$, Nguyen Van Sinh ${ }^{2}$ \\ ${ }^{1}$ Botanical Garden-Institute of Far Eastern Branch of the Russian Academy of Sciences, \\ Vladivostok, Russia \\ ${ }^{2}$ Institute of Ecology and Biological Resources, VAST
}

\begin{abstract}
The checklist of liverworts and hornworts of Vietnam was published more than 50 years ago. The aim of this study is to provide new checklist based on literature survey. The updated checklist includes 5 species of hornworts and 296 species of liverworts. The largest taxonomic group in regional hepatic flora is Lejeuneaceae that counts 178 species. It is estimated that the known taxonomic diversity will exceed 400 taxa once purposeful researches would be made.
\end{abstract}

Keywords: Anthocerotae, Hepaticae, checklist, hornworts, liverworts, Indochina, Vietnam.

Citation: Bakalin V., Nguyen Van Sinh, 2016. The checklist of liverworts (Hepaticae) and hornworts (Anthocerotae) of Vietnam updated based on literature survey. Tap chi Sinh hoc, 38(4): 480-491. DOI: $10.15625 / 0866-7160 / \mathrm{v} 38 \mathrm{n} 4.8771$.

*Corresponding author: vabakalin@gmail.com.

Received 10 October 2016, accepted 20 December 2016

\section{INTRODUCTION}

Vietnam and Thailand are two countries in Indochina those were estimated as the taxonomically richest in the region. Both of them have attracted attention of bryologists for a long time since the last quarter of ninetieth century. The first checklist of Vietnam bryophytes was published by a prominent Hungarian bryologist T. Pócs (1965). It includes 394 species of Musci and 162 species of Hepaticae and Anthocerotae. Since then, knowledge on hepatics in this area has been updated. After publication of the first checklist, intensive works were continued by T. Pócs, T. Ninh (former PhD student of Pócs and the first Vietnamese bryologist), P. Tixier and some other bryologists. The similar progress has been made on moss flora recognition and the new checklist of Vietnam mosses was published recently (Si \& Khang, 2012). Contrary to the mosses, the complete taxonomic list of hepatics was never published after Pócs" "prodrome" (1965). One of the current tasks for Vietnamese bryologists is, therefore, to publish newly updated checklist of liverworts and hornworts of Vietnam compiled in accordance with modern nomenclature.

\section{METHODS}

\section{List of taxa}

The list comprises alphabetically organized lists of hornworts and liverworts which were mentioned in the literature sources since (and including) "Prodrome de la bryoflore du Vietnam" (Pócs , 1965). The most of literature sources includes original data with specimen citations from the country. Besides some monographs providing citations for Vietnam were added. In total 45 sources were accumulated. Each species is annotated with references to the literature where the species was indicated. The nomenclature is standardized following to the "World checklist of hornworts and liverworts" (Söderström et al., 2016) with the exception for Plectocolea, which was treated as the genus separate from Solenostoma. Each citation is supplemented by the indication of heterotypic synonyms on which the record is based, although homotypic synonyms are omitted. The most of infraspecific taxa (like variety and forma), which status 
remains unclear are not mentioned.

\section{RESULTS}

\section{ANTHOCEROTOPHYTA}

Anthoceros subtilis Steph.: Pócs, 1965 (as A. brunneus Steph. and A. fuscus Steph.).

Folioceros fuciformis (Mont.) D. miyabenus Steph.).

Phaeoceros lamellisporus (Stephani) Udar \& D. K. Singh: Pócs, 1965 (as Anthoceros lamellisporus Steph., status unclear)

Phaeoceros carolinianus (Michx.) Prosk.: Pócs, 1965 (as P. communis (Steph.) Pócs and Anthoceros tonkinensis Steph.).

P. erectus Udar et D.K.Singh: Pócs, 1965.

\section{MARCHANTIOPHYTA}

Acrolejeunea fertilis (Reinw., Blume et Nees) Schiffn.: Gradstein, 1975; Pócs, 1965.

A. sandvicensis (Gottsche) Steph.: Manju et al., 2012; Pócs, 1965; Pócs, 1970; Pócs, 1971; Zhu \& So, 2001.

Acromastigum echinatiforme (De

Not.)

A.Evans: Pócs, 1971.

A. laevigatum A.Evans: Pócs, 1965.

Bazzania sandei Steph.: Pócs, 1965.

B. angustifolia Horik.: Pócs, 1968b; Pócs, 1969; Pócs et al., 1967.

B. angustistipula N.Kitag.: Pócs, $\quad 1969$ Pócs, 1971.

B. asperrima Steph.: Pócs, 1965. 1971.

B. asymmetrica (Steph.) N.Kitag.: Pócs,

B. fauriana (Steph.) S. Hatt.: Pócs, 1969; Pócs, 1971; Pócs et al., 1967; Pócs et al., 2013.

B. intermedia (Gottsche et Lindenb.) Trevis.: Pócs, 1965; Pócs, 1968b.

B. japonica (Sande Lac.) Lindb.: Pócs, 1969; Pócs \& Ninh, 2005.

B. praerupta (Reinw., Blume et Nees) Trevis.: Pócs, 1969; Pócs et al., 1967 (as B. yakushimensis Horik.).

B. recurva (Mont.) Trevis.: Pócs, 1965.
B. revoluta (Steph.) N.Kitag: Pócs, 1969 (as B. recurvolimbata (Steph.) N. Kitag.).

B. tridens (Reinw., Blume et Nees) Trevis.: Pócs, 1965 (also as B. oblonga (Mitt.) Schiffn.); Pócs, 1968b (as B. oblonga (Mitt.) Schiffn.); Pócs, 1969; Pócs, 1971; Pócs \& Ninh, 2005; Pócs et al., 2013.

- var. assamica (Steph.) Pócs: Pócs, 1969; Pócs, 1971.

- var. cornutistipula (Steph.) Pócs: Pócs, 1965 (as B. cornutistipula (Steph.) Pócs)

B. vietnamica Pócs: Pócs, 1969; Pócs, 1971.

Caudalejeunea reniloba (Gottsche) Steph.: Pócs, 1965 (as C. stephanii (Spruce) Steph.); Pócs \& Ninh, 2005 (as C. recurvistipula (Gott.) Schiffn.); Pócs \& Ninh, 2012.

Cheilolejeunea birmensis (Steph.) Mizut.: Pócs, 1971.

C. ceylanica (Gottsche) R.M.Schust. et Kachroo: Pócs, 1965; Pócs et al., 2013; Zhu \& So, 2001.

C. eximia (Jovet-Ast et Tixier) R.L.Zhu et M.L.So: Pócs, 1965; Zhu \& So, 2001.

C. falsinervis (Sande Lac.) R.M.Schust. et Kachroo: Pócs, 1965.

C. incisa (Gottsche) R.M.Schust. et Kachroo: Pócs, 1965.

C. insignis Jovet-Ast et Tixier: Pócs, 1965.

C. meyeniana (Nees, Lindenb. et Gottsche) R.M.Schust. et Kachroo: Pócs, 1965.

C. ryukyuensis Mizut.: Pócs \& Ninh, 2005.

C. streimannii Pócs et Ninh: Pócs \& Lee, 2016; Pócs \& Ninh, 2005.

C. trapezia (Nees) Kachroo et R.M.Schust.: Pócs, 1965 (as C. longiloba (Steph.) Kachroo et Schust.); Pócs, 1970 (as C. subplanilobula Chen et Wu); Pócs et al., 2013; Zhu \& So, 2001 (as $C$. imbricata (Steph.) S. Hatt.).

C. trifaria (Reinw., Blume et Nees) Mizut.: Pócs, 1965.

C. ventricosa (Schiffn. ex P.Syd.) Xiao L.He: Pócs et al., 2013.

C. viridis Steph.: Pócs, 1965.

C. xanthocarpa (Lehm. et Lindenb.) 
Malombe: Pócs, 1965.

Cololejeunea aequabilis (Sande

Lac.)

Schiffn.: Zhu \& So, 2001 (as C. yulensis (Steph.) Benedix).

C. albodentata P.C.Chen et P.C.Wu: Zhu \& So, 2001 .

C. amoena Benedix: Pócs et al., 2013; Tixier, 1970.

C. angustiflora (Steph.) Mizut.: Pócs \& Podani, 2015; Pócs et al., 2013.

C. appressa (A.Evans) Benedix: Pócs, 1965; Pócs et al., 2013; Tixier, 1970. 1985.

C. bichiana Tixier: Tixier, 1974; Tixier,

C. ceatocarpa (Ångstr.) Steph.: Pócs \& Lee, 2016; Pócs \& Ninh, 2012; Tixier, 1985.

C. ceratilobula (P.C.Chen) R.M.Schust.: Manju et al., 2012; Pócs, 1971; Pócs \& Ninh, 2005; Pócs et al., 1967 (as Pedinolejeunea formosana (Mizut.) P.C. Chen \& P.C. Wu); Tixier, 1970 (as Cololejeunea formosana Mizut.); Zhu \& So, 2001.

C. chenii Tixier: Pócs, 2012; Tixier, 1985; Zhu \& So, 2001.

C. chrysanthemi Tixier: Tixier, 1974.

C. cordiflora Steph.: Pócs \& Ninh, 2012; Tixier, 1985.

C. dankiaensis Tixier: Chantanaorrapint \& Pócs, 2014.

C. desciscens Steph.: Zhu \& So, 2001.

C. diaphana A.Evans: Pócs \& Bernecker, 2009 (as C. ninhbinhiana Tixier); Pócs et al., 2013; Tixier, 1974 (as C. ninhbinhiana Tixier).

C. elephantorum Tixier: Tixier, 1985.

C. ensifera Tixier: Chantanaorrapint \& Pócs, 2014.

C. equialbi Tixier: Pócs \& Podani, 2015; Tixier, 1985; Zhu \& So, 2001.

C. falcata (Horik.) Benedix: Pócs, 1965 (as C. falcatoides Benedix); Tixier, 1970 (as $C$. falcatoides Benedix); Zhu \& So, 2001.

C. filicis (Herzog) Piippo: Pócs \& Ninh, 2005; Tixier, 1974 (as C. pocsii Tixier); Tixier, 1985 (as C. pocsii Tixier); Zhu \& So, 2001.
C. floccosa (Lehm. et Lindenb.) Schiffn.. Pócs, 1965; Pócs et al., 2013; Tixier, 1970; Zhu \& So, 2001.

- var. aurita Benedix: Pócs \& Ninh, 2012.

C. fusca (Steph.) Mizut.: Pócs, 1965 (as Physocolea balansae Steph.); Tixier, 1985 (as Cololejeunea balansae (Steph.) Mizut.); Zhu \& So, 2001.

C. gottschei (Steph.) Pandé, K.P.Srivast. et Ahmad: Pócs, 1971 (as C. yunnanensis (Chen) Pócs); Pócs \& Ninh, 2005; Pócs \& Ninh, 2012; Zhu \& So, 2001.

C. grossepapillosa (Horik.) N.Kitag.: Pócs, 1970.

C. grushvitzkiana Pócs: Pócs, 1971.

C. gynophthalma Benedix: Pócs, 1965.

C. haskarliana (Lehm.) Schiffn.: Pócs \& Ninh, 2012; Pócs et al., 2013; Tixier, 1985; Zhu \& So, 2001. 1985.

C. hildebrandii (Austin) Steph.: Tixier,

C. hirta Steph.: Tixier, 1985.

C. hoabinhiana Tixier: Tixier, 1974; Tixier, 1985.

C. horikawana (S.Hatt.) Mizut.: Zhu \& So, 1999.

C. hungii Tixier: Tixier, 1974; Tixier, 1985.

C. indosinica Tixier: Zhu \& So, 2001.

C. inflata Steph: Pócs, 1965 (as C. oshimensis (Horik.) Benedix); Pócs, 2012; Pócs \& Ninh, 2005; Pócs et al., 2013; Zhu \& So, 2001.

C. inflectens (Mitt.) Benedix: Pócs, 1965 (as C. ciliatilobula Schiffn.); Pócs et al., 2013; Zhu \& So, 2001.

C. lanciloba Steph.: Pócs, 1965 (also as Leptocolea tonkinensis Steph.); Pócs \& Ninh, 2005; Pócs \& Ninh, 2012; Pócs et al., 2013; Tixier, 1985 (as Cololejeunea bolombensis (Steph.) Vaden Berghen).

C. latilobula (Herzog) Tixier: Manju et al., 2012; Pócs \& Ninh, 2012; Tixier, 1985; Zhu \& So, 2001.

C. littoralis Tixier: Tixier, 1985.

C. longifolia (Mitt.) Benedix ex Mizut.: 
Pócs, 1965 (as Physocolea acinacifolia Steph.); Pócs, 2012; Pócs \& Ninh, 2012.

C. macounii (Spruce) A.Evans: Tixier, 1985; Zhu \& So, 2001.

C. madothecoides (Steph.)

Benedix:

Chantanaorrapint \& Pócs, 2014; Pócs \& Ninh, 2005; Tixier, 1985.

C. mutabilis Benedix: Pócs, 1965.

C. obliqua (Nees et Mont.) Schiffn.: Pócs, 1965 (as C. nymanii (Steph.) Benedix); Pócs, 1971 (as C. goebelii (Gott. ex Schiffn.) Schiffn.); Tixier, 1985 (as C. goebelii (Gott.) Schiffn. and C. scabrifolia (Gott.) Schiffn.).

C. ocellata (Horik.) Benedix: Pócs \& Lee, 2016; Zhu \& So, 2001.

C. ocelloides (Horik.) Mizut.: Pócs, 1965 (as C. leonidens Benedix); Pócs, 1971 (as C. leonidens Benedix); Zhu \& So, 2001. 1985.

C. ombrophila Tixier: Tixier, 1970; Tixier, 1985.

C. papillosa (K.I.Goebel) Mizut.: Tixier,

C. peraffinis (Schiffn.) Schiffn.: Pócs, 1968b; Zhu \& So, 2001.

C. plagiophylla Benedix: Tixier, 1985.

C. planiflora Benedix: Pócs, 1968b.

C. planissima (Mitt.) Abeyw.: Pócs \& Ninh, 2005; Pócs \& Ninh, 2012; Tixier, 1985. 1998.

C. platyneura (Spruce) A.Evans: Zhu \& So,

C. pseudofloccosa (Horik.) Benedix: Pócs, 1965; Pócs, 2012.

C. pseudoschmidtii Tixier: Tixier, 1985; Zhu \& So, 2001.

C. pseudostephanii Tixier: Pócs \& Lee, 2016; Pócs et al., 2013.

C. raduliloba Steph.: Manju et al., 2012; Tixier, 1985; Zhu \& So, 2001.

C. reineckeana Steph.: Tixier, 1985. 2001.

C. schmidtii Steph.: Pócs, 1965; Zhu \& So,

C. selangorensis Tixier: Tixier, 1985.
C. serrata (Steph.) Benedix: Pócs, 1965; Tixier, 1985.

C. serrulata Steph.: Zhu \& So, 2001.

C. sigmoidea Jovet-Ast et Tixier: Pócs, 1965; Pócs et al., 2013; Tixier, 1970; Tixier, 1985; Zhu \& So, 2001.

C. sintenisii (Steph.) Pócs: Pócs et al., 2013.

C. spathulifolia (Steph.)

H.A.Mill.:

Chantanaorrapint \& Pócs, 2014.

C. sphaerodonta Mizut.: Pócs et al., 2013; Zhu \& So, 2001.

C. streimannii Pócs: Pócs \& Piippo, 2011.

C. stylilobula Tixier: Tixier, 1985.

C. stylosa Steph.: Tixier, 1985; Zhu \& So, 2001.

C. tenella Benedix: Tixier, 1970; Tixier, 1985; Zhu \& So, 2001.

C. teurnoumensis Tixier: Tixier, 1985.

C. tranninhiana Tixier: Pócs \& Podani, 2015; Tixier, 1974; Tixier, 1985.

C. trichomanis (Gottsche) Besch.: Pócs, 1965; Pócs \& Ninh, 2005; Tixier, 1985 (as $C$. dalatensis Tixier).

C. verrucosa Steph.: Pócs, 1965; Pócs, 2012; Pócs \& Ninh, 2012; Pócs et al., 2013; Tixier, 1985; Zhu \& So, 2001.

C. vietnamensis Tixier: Tixier, 1985.

Colura acroloba (Prantl) Jovet-Ast: Pócs, 1965; Pócs et al., 2013; Zhu \& So, 2001.

C. ari (Steph.) Steph.: Pócs, 2013; Zhu \& So, 2001.

C. brevistyla Herzog: Pócs \& Ninh, 2005.

C. conica (Sande Lac.) K.I.Goebel: Pócs, 1965 (as C. acutifolia S. Jovet-Ast); Pócs, 2013; Pócs \& Ninh, 2012; Zhu \& So, 2001.

C. corniantha Grolle: Pócs, 1965 (as C. cornuta Jovet-Ast n. inval.)

C. corynophora (Nees, Lindenb. et Gottsche) Trevis.: Pócs, 1965; Pócs \& Ninh, 2005; Tixier, 1970.

C. denticulata Jovet-Ast: Pócs, 1965.

C. inflata K.I.Goebel: Pócs, 1965. 
C. jovet-astiae Grolle: Pócs, 1965 (as C. undulata Jovet-Ast).

C. karstenii K.I.Goebel: Pócs, 1965; Pócs, 2013; Zhu \& So, 2001.

C. ornata K.I.Goebel: Pócs \& Ninh, 2012.

C. superba (Mont.) Steph.: Pócs, 1965; Pócs, 2013; Pócs et al., 2013. 1965.

C. tenuicornis (A.Evans) Steph.: Pócs,

C. tixieri Jovet-Ast: Pócs, 1965; Tixier, 1970.

C. valida Jovet-Ast: Pócs, 1965.

C. vietnamensis Jovet-Ast et Tixier: Pócs, 1965.

Cyathodium aureonitens (Griff.) Mitten. Srivastana \& Dixit, 1996.

C. smaragdinum Schiffn.: Pócs, 1965 (as $C$. balansae Steph.).

Diplasiolejeunea cavifolia Steph.: Pócs, 1965 (as D. javanica Steph.).

D. cobrensis Steph.: Pócs, 1965 (as D. incurvata Jovet-Ast et Tixier); SchäferVerwimp, 2006.

D. rudolphiana Steph.: Pócs, 1965; Pócs \& Lee, 2016; Schäfer-Verwimp, 2006; Zhu \& So, 2001.

Drepanolejeunea angustifolia (Mitt.)

Grolle: Manju et al., 2012; Pócs, 1965 (as $D$. tenuis (Nees) Schiffn.); Pócs, 1968b (as D. tenuis (Nees) Schiffn.); Pócs, 1971 (as D. tenuis (Nees) Schiffn.); Pócs \& Ninh, 2012; Pócs et al., 2013; Zhu \& So, 2001.

D. bidoupensis Pócs: Pócs et al., 2013.

D. commutata Grolle et R.L.Zhu: Pócs et al., 2013; Zhu \& So, 2001.

D. dactylophora (Nees, Lindenb. et Gottsche) J.B.Jack et Steph.: Pócs, 1965; Pócs et al., 2013; Zhu \& So, 2001.

D. elegans Herzog: Pócs, 1965.

D. erecta (Steph.) Mizut.: Pócs, 1971 (as D. japonica Horik.); Zhu \& So, 2001.

D. fleischeri (Steph.) Grolle et R.L.Zhu: Pócs et al., 2013.

D. foliicola Horik.: Pócs et al., 2013.
D. nymanii Steph.: Pócs et al., 2013.

D. obliqua Steph.: Pócs et al., 1967.

D. pentadactyla (Mont.) Steph.: Pócs, 1965 (as D. micholitzii Steph.); Pócs \& Ninh, 2012; Pócs et al., 2013.

D. spicata (Steph.) Grolle et R.L.Zhu: Pócs, 1965; Pócs et al., 2013; Zhu \& So, 2001. 1970.

D. tenera K.I.Goebel: Pócs, 1965; Pócs,

D. ternatensis (Gottsche) Schiffn.: Pócs, 1970. 1965.

D. teysmannii (Gottsche) Steph.: Pócs,

D. thwaitesiana (Mitt.) Steph.: Pócs, 1965; Pócs et al., 2013; Zhu \& So, 2001.

D. tricornua Herzog: Pócs et al., 2013.

D. vesiculosa (Mitt.) Steph.: Pócs, 1965; Zhu \& So, 2001.

D. yunnanensis (P.C.Chen) Grolle et R.L.Zhu: Kumar et al, 2015; Pócs, 2012; Pócs et al., 1967; Pócs et al., 2013.

Dumortiera hirsuta (Sw.) Nees: Pócs, 1965 (as D. trichocephala (Hook.) Nees).

- subsp. nepalensis (Taylor) R.M.Schust.: Pócs \& Ninh, 2005.

Frullania alstonii Verd.: Pócs, 1971; Pócs \& Ninh, 2005; Pócs et al., 2013.

$F$. apiculata (Reinw., Blume et Nees) Nees: Pócs, 1965 (also as F. anamensis Steph.); Pócs \& Ninh, 2005; Pócs et al., 2013.

F. campanulata Sande Lac.: Pócs, 1965.

F. densiloba Steph. ex A.Evans: Pócs, 1965.

F. ericoides (Nees) Mont.: Pócs, 1965 (as $F$. squarrosa Nees and $F$. lacinioisa Lehm.).

F. gracilis (Reinw., Blume et Nees) Nees: Pócs, 1965.

F. hamatiloba Steph.: Pócs \& Ninh, 2005.

F. iwatsukii S.Hatt.: Hattori, 1976. 2005.

F. junghuhniana Gottsche: Pócs \& Ninh,

F. meyeniana Lindenb.: Pócs, 1965.

F. moniliata (Reinw., Blume et Nees) Mont.: Pócs, 1965 (also as F. balansae Steph.); 
Pócs, 1971.

F. monocera (Hook.f. et Taylor) Gottsche: Pócs, 1965 (as $F$. hampeana Nees).

F. nepalensis (Spreng.) Lehm. et Lindenb.: Pócs, 1965; Pócs et al., 1967 (as F. nishiyamensis Steph.)

F. neurota Taylor: Pócs, 1965.

F. nodulosa (Reinw., Blume et Nees) Nees: Hattori, 1980.

F. orientalis Sande Lac.: Hattori, 1975.

F. physantha Mitt.: Pócs, 1968b; Pócs et al., 1967; Thaithong \& Hattori, 1977.

$F$. pocsantha Thaithong et S.Hatt.: Thaithong \& Hattori, 1977

F. ramuligera (Nees) Mont.: Pócs, 1971; Pócs et al., 1967. 1965.

F. rio-janeirensis (Raddi) Ångstr.: Pócs,

F. serrata Gottsche: Pócs, 1971.

F. ternatensis Gottsche: Pócs, 1965.

Fuscocephaloziopsis connivens subsp. fissa (Steph.) Váňa et L.Söderstr.: Pócs \& Ninh, 2005.

Herbertus armitanus (Steph.) H. A. Mill.: Juslén, 2006.

H. dicranus (Gottsche, Lindenb. et Nees) Trevis.: Juslén, 2006; Pócs, 1965 (under H. chinensis); Pócs, 1971 (as H. chinensis Steph.).

H. longifissus Steph.: Pócs, 1965.

H. ramosus (Steph.) H. A. Mill.: Juslén, 2006; Pócs, 1970.

Heteroscyphus argutus (Reinw., Blume et Nees) Schiffn.: Piippo, 1993; Pócs, 1965; Pócs, 1971; Pócs \& Ninh, 2005.

H. coalitus (Hook.) Schiffn.: Pippo, 1993; Pócs, 1965 (as H. communis (Steph.) Schiffn.); Pócs, 1968b (as H. communis (Steph.) Schiffn.); Pócs, 1971 (as H. communis (Steph.) Schiffn.).

H. splendens (Lehm. et Lindenb.) Grolle: Pócs, 1971 (as H. decurrens (Nees) Schiffn.).

H. zollingeri (Gottsche) Schiffn.: Pócs \& Ninh, 2005.

Isotachis japonica Steph.: Pócs et al., 1967.
Jubula hutchinsiae subsp. javanica (Steph.) Verd.: Pócs, 1965 (as J. tonkiensis Steph.).

Lejeunea alata Gottsche: Pócs, 1965 (as Taxilejeunea mitracalyx Eifr.); Zhu \& So, 2001

L. anisophylla Mont.: Pócs, 1971 (as L. boninensis Horik.); Pócs \& Ninh, 2005; Pócs \& Ninh, 2012; Zhu \& So, 2001.

L. apiculata Sande Lac.: Pócs, 1965 (as Drepanolejeunea formosana Horik.); Pócs et al., 2013; Zhu \& So, 2001.

L. exilis (Reinw., Blume et Nees) Grolle: Pócs \& Ninh, 2005.

L. flava (Sw.) Nees: Pócs, 1965; Pócs et al., 2013.

L. lunatigastria Tixier: Tixier, 1974.

L. micholitzii Mizut.: Pócs \& Ninh, 2005.

L. obscura Mitt.: Pócs \& Ninh, 2005.

L. papilionacea Prantl: Pócs, 1965 (as L. infestans Steph.).

L. subolivacea Mizut.: Pócs, 1965 (as L. olivacea Steph.).

Lejeunea trinitensis Lindenb.: Pócs, 1965 (as Stylolejeunea asiatica Jovet-Ast et Tixier).

L. umbilicata (Nees) Nees: Pócs, 1965.

Lepidolejeunea bidentula (Steph.)

R.M.Schust.: Piippo, 1986; Pócs, 1965 (also as Pycnolejeunea corticola Steph.); Tixier, 1970 (as P. bidentula Steph.); Zhu \& So, 2001.

Lepidozia tonkinensis Steph.: Pócs, 1965 (status unclear, nomen nudum).

L. trichodes (Reinw., Blume et Nees) Nees: Pócs, 1965; Pócs, 1968b; Pócs \& Ninh, 2005.

Leptolejeunea apiculata (Horik.) S.Hatt.: Pócs \& Lee, 2016; Pócs et al., 2013.

L. balansae Steph.: Manju et al., 2012; Pócs, 1965; Pócs \& Ninh, 2012; Pócs et al., 2013; Zhu \& So, 2001.

L. elliptica (Lehm. et Lindenb.) Besch.: Pócs, 1965 (also as L. subacuta Steph.); Pócs, 1970 (as L. subacuta Steph.); Pócs \& Ninh, 2005; Pócs \& Ninh, 2012; Pócs et al., 2013; Tixier, 1970 (as L. subacuta Steph.).

L. epiphylla (Mitt.) Steph.: Pócs \& Ninh, 2012. 
L. foliicola Steph.: Pócs, 1965.

L. maculata (Mitt.) Schiffn.: Pócs \& Ninh, 2012; Pócs et al., 2013.

L. radiata Steph.: Pócs, 1965.

L. serrulata Herzog: Pócs, 1965; Pócs \& Podani, 2015.

L. subdentata Schiffn. ex Herzog: Pócs, 1965; Pócs et al., 2013; Zhu \& So, 2001.

L. vitrea (Nees) Schiffn.: Pócs, 1965.

Lopholejeunea applanata (Reinw., Blume et Nees) Schiffn.: Pócs \& Ninh, 2005.

L. eulopha (Taylor) Schiffn.: Pócs \& Ninh, 2005; Pócs et al., 2013; Zhu \& Gradstein, 2005.

L. nigricans (Lindenb.) Schiffn.: Zhu \& Gradstein, 2005.

L. subfusca (Nees) Schiffn.: Pócs, 1965; Pócs \& Ninh, 2005; Zhu \& Gradstein, 2005.

L. vietnamica Tixier: Tixier, 1974 (probably another genus, cf. Söderström et al., 2016).

Marchantia emarginata Reinw., Blume et Nees: Bischler-Causse, 1989; Pócs, 1965 (as M. palmata Nees).

- subsp. tosana (Steph.) Bischl.: BischlerCausse, 1989; Pócs, 1965 (as M. angusta Steph.); Pócs \& Ninh, 2005.

M. paleacea Bertol.: Bischler-Causse, 1989; Pócs, 1965.

M. polymorpha subsp. ruderalis Bischl. et Boissel.-Dub.: Bischler-Causse, 1989.

Mastigolejeunea humilis (Gottsche)

Schiffn.: Pócs, 1965.

M. recondita (Steph.) Mizut.- Pócs, 1965 (as Ptychanthus reconditus Nees).

Mastigophora diclados (Brid. ex F.Weber) Nees: Pócs, 1965 (also as M. gracillima Steph.); Pócs, 1971 (as M. gracillima Steph.); Pócs et al., 2013 (as M. gracillima Steph.).

Metalejeunea cucullata (Reinw., Blume et Nees) Grolle: Pócs, 1965 (also as Microlejeunea cumingiana Steph.); Pócs, 1971; Pócs et al., 2013.

Metzgeria conjugata Lindb. ssp. japonica (Hatt.) Kuwah.: Pócs, 1968b; Pócs, 1971; Pócs \& Ninh, 2005.
M. consanguinea Schiffn.: So, 2003.

M. furcata (L.) Corda: Pócs, 1965; Pócs et al., 2013.

M. pubescens (Schrank) Raddi: Pócs, 1968b.

Microlejeunea punctiformis (Taylor) Steph.: Manju et al., 2012; Pócs, 1968b; Pócs, 1971; Pócs \& Ninh, 2005; Zhu \& So, 2001.

Myriocoleopsis vuquangensis (Pócs et Ninh) Pócs: Pócs, 2010; Pócs \& Ninh, 2005.

Notoscyphus lutescens (Lehm. et Lindenb.) Mitt.: Pócs, 1965 (as Prasanthus paroicus (Schiffn.) Kamim.).

Otolejeunea hoana (Tixier) Grolle: Tixier, 1980.

O. semperiana (Steph.) Grolle: Pócs, 1965.

Plagiochasma appendiculatum Lehm. et Lindenb.: Pócs, 1965 (as $P$. reboulioides Horik.).

Plagiochila bantamensis (Reinw., Blume et Nees) Mont.: Pócs, 1970 (as P. meyeniana Steph.); So, 2001.

P. chinensis Steph.: Pócs, 1970 (also as $P$. wilsoniana Steph.); So, 2001.

P. durelii Schiffn: Inoue, 1968 (as $P$. vietnamica $\mathrm{H}$. Inoue); So, 2001.

P. flexuosa Mitt.: Pócs, 1968b; So, 2001.

P. fordiana Steph.: Inoue, 1968; So, 2001.

P. fruticosa Mitt.: Pócs, 1965; Pócs, 1968b; Pócs, 1970; Pócs, 1971; So, 2001.

P. furcifolia Mitt.: Inoue, 1968 (as $P$. fissifolia Steph.); Pócs, 1965 (as P. fissifolia Steph.); So, 2001.

P. grollei Inoue: Pócs, 1970; So, 2001. 2005.

P. integrilobula Schiffn.: Pócs \& Ninh,

P. javanica (Sw.) Nees et Mont.: Pócs \& Ninh, 2005.

P. junghuhniana Sande Lac.: So, 2001.

P. nepalensis Lindenb.: Pócs, 1970 (also as P. gollanii Steph.); So, 2001.

P. oblonga Inoue: Pócs, 1968b; Pócs, 1970.

P. obtusa Lindenb.: Pócs, 1965 (as $P$. eberhardtii Steph.); So, 2001. 
P. parvifolia Lindenb.: Pócs, 1965; Pócs, 1970 (also as $P$. consociata Steph. and $P$. ventricosa Steph.); So, 2001.

P. peculiaris Schiffn.: Pócs, 1970 (as $P$. crassitexta Steph.); So, 2001.

P. pseudorenitens Schiffn.: So, 2001.

P. pulcherrima Horik.: So, 2001.

P. renitens (Nees) Lindenb.: Pócs, 1971.

P. sandei Dozy ex Sande Lac.: Pócs, 1965.

P. sciophila Nees: Inoue, 1968 (as $P$. subplanata H. Inoue); Pócs, 1965 (as $P$. tonkiensis Steph.); Pócs, 1970 (also as $P$. subplanata H. Inoue); Pócs \& Ninh, 2005; So, 2001.

P. secretifolia Mitt.: So, 2001.

P. semidecurrens (Lehm. et Lindenb.) Lindenb.: Pócs, 1968b; Pócs, 1971; So, 2001.

P. subplana Lindenb.: Pócs, 1971.

P. trabeculata Steph.: Inoue, 1968 (as $P$. pocsii $\mathrm{H}$. Inoue); So, 2001.

Plagiochilion oppositum (Reinw., Blume et Nees) S.Hatt.: Pócs, 1965.

Plectocolea ariadne (Taylor ex Lehm.) Mitt.: Váňa, 1972. 1969.

Pleurozia gigantea (F.Weber) Lindb.: Pócs,

Plicanthus birmensis (Steph.) R.M.Schust.: Pócs, 1970; Pócs, 1971.

P. hirtellus (F.Weber) R.M.Schust.,: Pócs, 1965; Pócs, 1971.

Porella acutifolia (Lehm. et Lindenb.) Trevis.: Pócs, 1968a.

P. caespitans var. cordifolia (Steph.) S.Hatt. ex T.Katag. et T.Yamag.- Pócs, 1965 (as Madotheca urophylla C. Massal and as Porella piligera (Steph.) Pócs); Pócs, 1968a (also as $P$. piligera (Steph.) Pócs); Pócs, 1968b (as $P$. piligera (Steph.) Pócs). 1968 a.

- var. reflexigastria (Pócs) S.Hatt.: Pócs,

P. campylophylla (Lehm. et Lindenb.) Trevis.: Hattori, 1978.

P. densifolia (Steph.) S.Hatt.: Hattori, 1978; Pócs, 1968a.

P. grandifolia (Steph.) S.Hatt.: Hattori, 1978; Pócs, 1968a.

P. japonica (Sande Lac.) Mitt.: Hattori, 1978; Pócs, 1968a.

P. madagascariensis (Nees et Mont.) Trevis.: Pócs, 1968a.

P. obtusata (Taylor) Trevis.: Pócs, 1968a (as P. thuja (Dicks) Moore).

P. perrottetiana (Mont.) Trevis.: Hattori, 1978; Pócs, 1968a; Pócs, 1971.

P. plumosa (Mitt.) Parihar: Hattori, 1978; Pócs, 1968a; Pócs, 1968b.

P. striatus (Lehm. et Lindenb.) Nees: Pócs, 1965 (also as P. pyriformis Steph.); Pócs, 1970; Pócs, 1971; Pócs et al., 2013.

Pycnolejeunea convexifolia (Mitt.) Steph.: Pócs, 1965 (as Cheilolejeunea imbricata (Nees) S.Hatt.)

Radula acuminata Steph.: Pócs, 1965; Pócs, 1971; Pócs \& Ninh, 2005; Pócs et al., 2013; Yamada, 1979; Zhu \& So, 2001.

$R$. aneurismalis (Hook.f. et Taylor) Gottsche, Lindenb. et Nees: Pócs, 1971.

R. assamica Steph.: Pócs, 1965; Pócs, 1971; Pócs \& Ninh, 2005; Pócs et al., 2013; Yamada, 1979; Zhu \& So, 2001. 1979.

R. borneensis Steph.: Pócs, 1965; Yamada,

R. cavifolia Hampe ex Gottsche: Pócs et al., 2013; Yamada, 1979; Zhu \& So, 2001.

$R$. gedena Gottsche: Pócs et al., 2013.

R. javanica Gottsche: Pócs et al., 2013; Yamada, 1979. 1979.

R. nymannii Steph.: Pócs, 1965; Yamada,

R. obtusiloba Steph.: Pócs, 1971.

R. tjibodensis K.I.Goebel: Pócs, 1965; Zhu \& So, 2001.

Riccia crystallina L.: Pócs, 1965.

R. frostii Austin: Pócs, 1965 (as $R$. microspora Steph.). 2001.

Scapania ciliata Sande Lac.: Potemkin, 
Schiffneriolejeunea

Gradst.: Pócs, 1965.

Schistochila acuminata Steph.: Pócs, 1965 (as $S$. rigidula Horik.).

S. aligera (Nees et Blume) J.B.Jack et Steph.: Pócs, 1965 (also as S. philippinensis (Mont.) Jack. et Steph.); Pócs, 1968b; Pócs, 1971 (also as S. recurvata Buch.).

S. blumei (Nees) Trevis.: Pócs, 1971. 1965.

S. caudata R.M.Schust. et J.J.Engel: Pócs,

Spruceanthus semirepandus (Nees) Verd.: Pócs, 1965; Pócs, 1971.

S. sulcatus (Nees) Gradst: Pócs, 1965.

Tricholepidozia neesii (Lindenb.) E.D. Cooper: Pócs, 1971 (as Telaranea neesi (Lindenb.) Fulford).

Thysananthus comosus Lindenb.: Pócs et al., 1967.

T. spathulistipus (Reinw., Blume et Nees) Lindenb.: Pócs, 1965; Tixier, 1970.

Trichocolea pluma (Reinw., Blume et Nees) Mont.: Pócs, 1965.

T. tomentella (Ehrh.) Dumort.: Pócs, 1965.

Tuyamaella angulistipa (Steph.)

R.M.Schust. et Kachroo: Pócs, 1965.

T. molischii (Schiffn.) S.Hatt.: Pócs, 1965; Pócs et al., 2013; Zhu \& So, 2001.

Tylimanthus tonkinensis Steph: Pócs, 1965 (status unclear, nomen nudum)

\section{DISCUSSION}

The compiled checklist consists of 5 species of hornworts, including Phaeoceros lamellisporus of unclear status, and 296 species, 2 subspecies and 4 varieties of liverworts, including two nomen nudum (Lepidozia tonkinensis, Tylimanthus tonkinensis) with unknown types. More than the half of the known liverwort diversity comprised by Lejeuneaceae that count 178 species in the country. The subsequent families are quite small in taxa (Plagiochilaceae: 26 species and Frulaniaceae: 22).
As the result of researches of the last 50 years since the publication of the checklist by Pócs (1965), 141 species have been added to the liverwort and hornwort flora of Vietnam. Nevertheless, the known number of hepatic taxa is not comprehensive and the progress in the knowledge of taxonomic diversity of Vietnam is hardly near to the end. The liverwort and hornwort checklist of Thailand (Lai et al., 2008) includes 386 taxa that still is not the final number. Very preliminary we may estimate that the expected taxonomic diversity of Vietnam liverworts and hornworts exceeds 400 species and several taxa are needed to be described as new for science in the course of further researches.

Acknowledgements: The authors are deeply indebted to Dr. T. Pócs for his help with literature and some advices and also to Prof. R. Gradstein for some nomenclatural remarks. The study was partly supported by the collaboration program between Vietnam Academy of Science and Technology and Far Eastern Branch of the Russian Academy of Sciences (no. BAHT17002 and "Patterns of altitudinal zonation of hepatics in East and South-East Asia": VAST).

\section{REFERENCES}

Bischler-Causse H., 1989. Marchantia L. The Asiatic and Oceanic taxa. Bryophytorum Bibliotheca. Band 38. Cramer, BerlinStuttgart, pp. 1-317.

Chantanaorrapint S., Pócs T., 2014. Southern Thailand bryophytes I, with description of Cololejeunea ramromensis. In: Telnov D. (ed.) 2014: Biodiversity, Biogeography and Nature Conservation in Wallacea and New Guinea, II: 113-122.

Gradtsein S.R., 1975. A taxonomic Monograph of the Genus Acrolejeunea (Hepaticae). Bryophytorum Bibliotheca. Band 4. Cramer, Vaduz, 162 pp. +24 tables.

Hattori S., 1975. Notes on the Asiatic species of the genus Frullania, Hepaticae. VII. Journal Hattori Bot. Lab., 39: 277-313.

Hattori S., 1976. A new subspecies of Frullania 
iwatsuki from North Vietnam, Misc. Bryologica et Lichenologica, 7(5): 87-89.

Hattori S., 1978 Studies on the Asiatic species of the genus Porella (Hepaticae). VII. A synopsis of Asiatic Porellaceae. Journal Hattori Bot. Lab., 44: 91-120.

Hattori S., 1980. A revision of the subgenus Homotropantha of the genus Frullania, Hepaticae. Journal Hattori Bot. Lab., 47: 165-236.

He S., Nguyen S. K., 2012. New records and an updated checklist of the mosses of Vietnam. Tropical Bryology, 34: 32-88.

Inoue H., 1968. Contribution to the knowledge of the Plagiochilaceae of Southeastern Asia. XI. New or little known species of Plagiochila from North Vietnam. Journal Hattori Bot. Lab., 31: 297-311.

Juslén A., 2006. Revision of Asian Herbertus (Herbertaceae,

Marchantiophyta) Ann. Bot. Fennici, 43: 409-436.

Kumar S., Pocs T., Singh S. K., 2015. Two Drepanolejeunea [Marchantiophyta] New to Bryoflora of Meghalaya. Indian Journal of Forestry, 38(4) 343-349.

Lai M. J., Zhu R. L., Chantanaorrapint S., 2008. Liverworts and hornworts of Thailand: an updated checklist and bryofloristic accounts. Ann. Bot. Fennici, 45: 321-341.

Manju C. N., Pócs T., Rajesh K. P., Prakashkumar R., 2012. Lejeuneaceae (Marchantiophyta) of the Western Ghats, India. Acta Biologica Plantarum Agriensis, 2: $127-147$.

Piippo S., 1986. A monograph of the genera Lepidolejeunea and Luteolejeunea (Lejeuneaceae, Hepaticae). Acta Bot. Fennica, 132: 1-69.

Piippo S., 1993. On taxonomy and nomenclature of SW Asiatic Geocalycaceae (Hepaticae). Ann. Bot. Fennici, 30: $195-$ 203.

Pócs T., 1965. Prodrome de la bryoflore du Vietnam. Az Egri Tanárképző Főiskola Tudományos Közleményei, 3: 453-495.
Pócs T., 1968. The genus Porella in Vietnam. Journal Hattori Bot. Lab., 31: 65-93.

Pócs T., 1968. Troisième contribution à la bryoflore du Nord Vietnam. Récoltes polonaises aux environs de Sapa. Fragmenta Floristica Geobotanica, 14: 495-504.

Pócs T., 1969. A short survey of the Bazzania of North Viet-Nam. Journal Hattori Bot. Lab., 32: 79-94.

Pócs T., 1970. Adatok Észak-Vietnam mohaflórájához. V. (Fifth contribution to the bryoflora of North Vietnam). Botanikai Közlemények, 56: 139-147.

Pócs T., 1971. Studies on the mountain bryoflora of the Ha-Giang province (Democratic Republic of Vietnam): Hepaticae. Botanicheskiy Zhurnal, 56: 670677.

Pócs T., 2010. Myriocoleopsis in Southeast Asia. Tropical Bryology, 31: 123-125.

Pócs T., 2012. New or little known epiphyllous liverworts, XVI. A small collection from Laos. Acta Biologica Plantarum Agriensis, 2: 5-10.

Pócs T., 2013. The genus Colura (Lejeuneaceae) in New Guinea and in the neighboring areas. Chenia. 11: 12-38.

Pócs T., Bernecker A., 2009. Overview of Aphanolejeunea (Jungermanniopsisda) after 25 years. Polish Bot. Journal, 54(1): 1-11.

Pocs T., Lee G.E., 2016. Data to the Malaysian liverwort flora, II Cryptogamie, Bryologie, 37 (1): 39-52.

Pócs T., Ninh T., 2005. Contribution to the bryoflora of Vietnam, VI. On the liverwort flora of $\mathrm{Vu}$ Quang Nature Reserve. Acta Botanica Hungarica, 47(1-2): 151-171.

Pócs T., Ninh T., 2012. New or little known epiphyllous liverworts, XVII. Records from the Cát Tiên National Park, southern Vietnam. Acta Biologica Plantarum Agriensis, 2: 11-19.

Pócs T., Piippo S., 2011. Bryophyte flora of the Huon Peninsula, Papua New Guinea. LXXIV. Cololejeunea (Lejeuneaceae, 
Hepaticae). Acta Bryolichenologica Asiatica, 4: 59-137.

Pocs T., Podani J., 2015. Southern Thailand Bryophytes II. Epiphylls from the PhangNga area. Acta Botanica Hungarica, 57(12): 183-198

Pócs T., Tixier P., Jovet -Ast S., 1967. Adatok Észak-Vietnam mohaflórájához. II. Seconde contribution a la Bryoflore du Nord Vietnam. Botanikai Közlemények, 54: 27-38.

Pocs T., Luong T.-T., Ho B.-C., 2013. New or little known epiphyllous liverworts, XVIII. Records from the Bidoup-Núi Bà National Park, Vietnam, with the description of Drepanolejeunea bidoupensis, sp. nov. Cryptogamie, Bryologie, 34 (3): 287-298.

Potemkin A.D., 2001. Evolution, phylogeny and classification of Scapaniaceae (Hepaticae). Doctoral thesis. Saint-Petersburg, pp. 1-338.

Schäfer-Verwimp A., 2006. A new species of Diplasiolejeunea (Lejeuneaceae, Jungermanniopsida) from Sumatra, and a key for the genus in Asia. Herzogia, 19: 239-24

So M. L., 2001. Plagiochila (Hepaticae, Plagiochilaceae) in China. Systematic Botany Monographs. Vol. 60, pp. 1-214.

So M. L., 2003. The genus Metzgeria (Hepaticae) in Asia. Journal Hattori Bot. Lab., 94: 159-177.

Söderström L., Hagborg A., Konrat M. von, Bartholomew-Began S., Bell D. et al., 2016: World checklist of hornworts and liverworts. PhytoKeys, 59: 1-828.

Srivastava S. C., Dixit R., 1996. The genus Cyathodium Kunze. Journal Hattori Bot. Lab., 80: 149-215.

Thaithong O., Hattori S., 1977. A new species of Frullania from North Vietnam and the Indian F. physantha Mitt. Bulletin National Science Museum, Ser. B., 3(4): 149-154.

Tixier P., 1970. Bryophytae indosinicae: Bryophytes from Thai Tenasserim. Natural History Bulletin of the Siam Society, 23 : 541-560.

Tixier P., 1974. Bryophytae Indosinicae. A propos de quelques especes nouvelles recoltees au Vietnam. Annales HistoricoNaturales Musei Nationalis Hungarici, 66: 87-100.

Tixier P., 1980. Deux nouveaux Genres de Lejeuneacees Otolejeunea Grolle \& P. Tx. et Allorgella P. Tx. Nova Hedwigia, 32: 607-622.

Tixier P., 1985. Contribution à la Connaissance des Cololejeunoideae. Bryophytorum Bibl., 27: 1-431.

Váňa J., 1972. Miscellaneous notes on the Asiatic Jungermannioideae II.: Journal Hattori Bot. Lab., 36: 57-74.

Yamada K., 1979. A revision of Asian taxa of Radula, Hepaticae. Journal Hattori Bot. Lab., 45: 201-322.

Zhu R. L., Gradstein S. R., 2005. Monograph of Lopholejeunea (Lejeuneaceae, Hepaticae) in Asia. Systematic Botany Monographs. Vol. 74. pp. 1-98.

Zhu R. L., So M. L., 1998. Notes on the taxonomy and distribution of Cololejeunea platyneura (Hepaticae, Lejeuneaceae). Ann. Bot. Fennici, 35(3): 229-232.

Zhu R. L., So M. L., 1999. New records of Cololejeunea (Lejeuneaceae, Hepaticae) for China and Vietnam. Bot. Bull. Acad. Sin., 40: 165-171.

Zhu R. L., So M. L., 2001 Epiphyllous liverworts of China. Nova Hedwigia Beiheft, 121. pp. 1-418. 


\title{
DANH LỤC CÁC LOÀI RÊU TẢN (Hepaticae) VÀ RÊU SỪNG (Anthocerotae) CỦA VIỆT NAM
}

\author{
Vadim Bakalin ${ }^{1}$, Nguyễn Văn Sinh ${ }^{2}$
}

${ }^{1}$ Viện Vườn thực vật Phân viện Viễn Đông, Viện Hàn lâm khoa học Nga, Vladivostok, CHLB Nga ${ }^{2}$ Viện Sinh thái và Tài nguyên sinh vật, Viện Hàn lâm Khoa học và Công nghệ Việt Nam

\section{TÓM TẮT}

Danh lục các loài Rêu tản (Hepaticae) và Rêu sừng (Anthocerotae) của Việt Nam được công bố cách đây hơn 50 năm. Bài báo này cung cấp một danh lục mới dựa trên nghiên cứu các tài liệu đã công bố. Danh lục cập nhật bao gồm 5 loài rêu sừng và 296 loài rêu tản. Nhóm taxon lớn nhất của khu hệ rêu tản là họ Lejeuneaceae bao gồm 178 loài. Theo đánh giá của chúng tôi, có đến hơn 400 loài Rêu tản và Rêu sừng ở Việt Nam.

Tù khóa: Danh lục, rêu tản, rêu sừng, Đông Dương, Việt Nam. 